\title{
May 2014 Imaging Case of the Month
}

\author{
Michael B. Gotway, MD \\ Department of Radiology \\ Mayo Clinic Arizona \\ Scottsdale, AZ
}

Clinical History: A 66-year-old woman presented with complaints of cough worsening over the previous several months. Her prior medical history was largely otherwise unremarkable. Frontal chest radiography (Figure 1) was performed for evaluation.

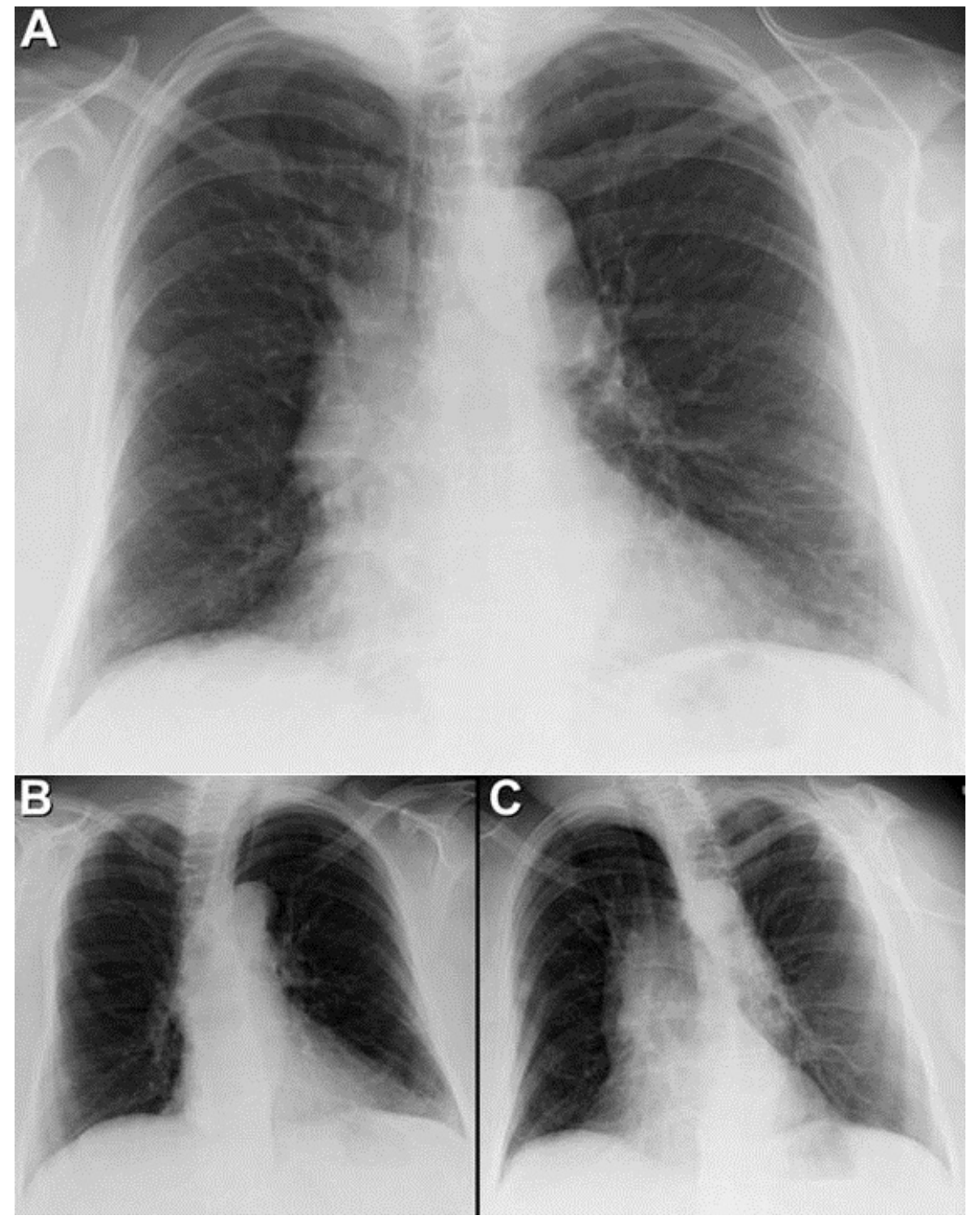

Figure 1. Panel A: Frontal chest radiograph. Panel B: Right anterior oblique image. Panel C: Left anterior oblique image. 
Which of the following statements regarding the chest radiograph is most accurate?

1. The chest radiograph shows a solitary pulmonary nodule

2. The chest radiograph shows multifocal airway thickening and bronchiectasis

3. The chest radiograph shows multifocal, bilateral cavitary nodules and consolidation

4. The chest radiograph shows multifocal, somewhat basal predominant linear opacities within diminished lung volumes, suggesting fibrosis

5. The chest radiograph shows multiple small nodules, suggesting a miliary pattern 


\section{Correct! \\ 1. The chest radiograph shows a solitary pulmonary nodule}

The frontal chest radiograph shows a borderline-to-mildly enlarged heart with a tortuous and possibly dilated ascending aorta (assessment is compromised by the left anterior oblique positioning of the chest radiograph). A circumscribed solitary pulmonary nodule is faintly visible over the peripheral right mid-lung (Figure 2).

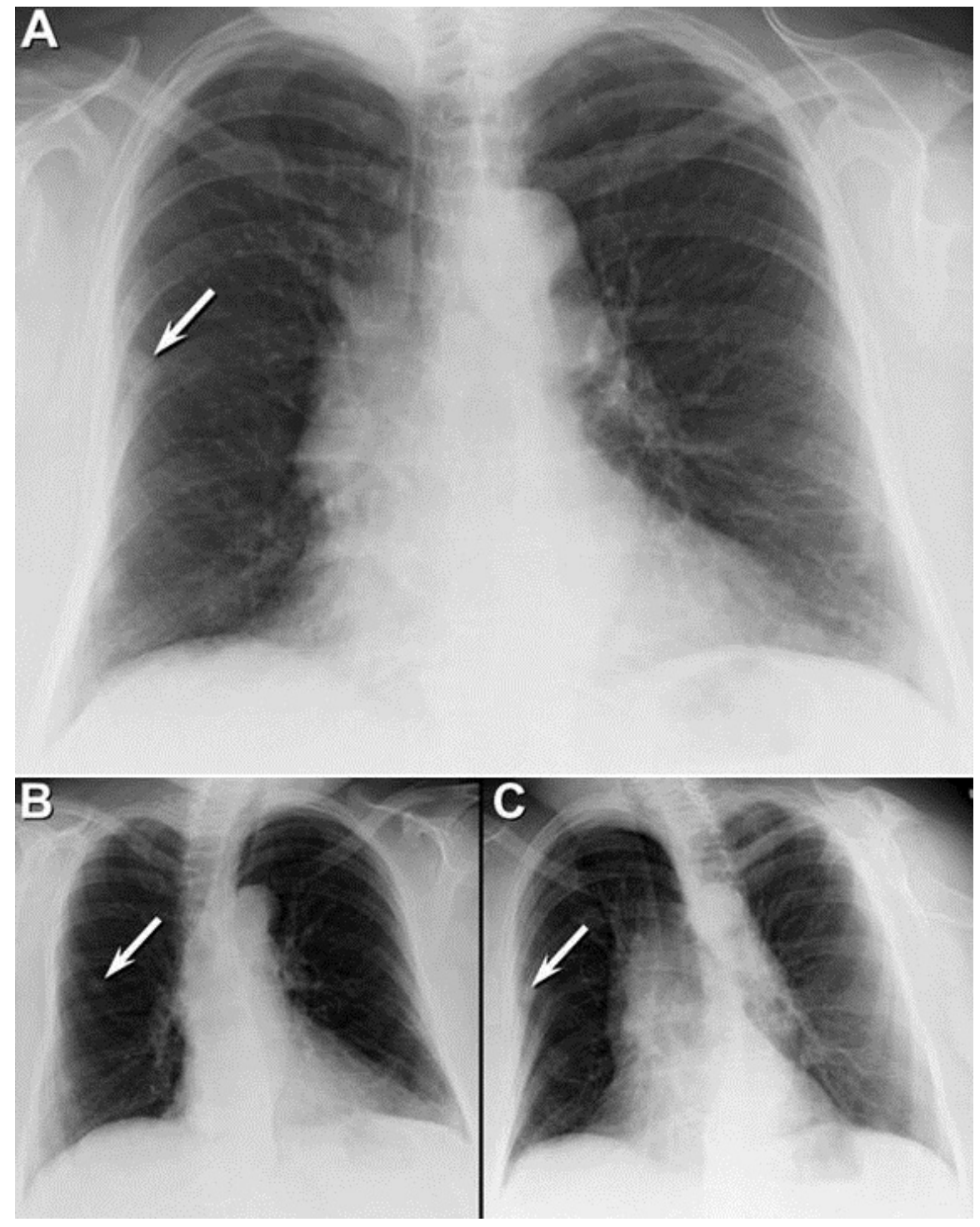

Figure 2. A circumscribed solitary pulmonary nodule (arrow) is faintly visible over the peripheral right mid-lung.

No convincing evidence of airway thickening or bronchiectasis is evident, and the lung volumes appear normal. No features of pulmonary fibrosis are seen. A small nodular, or miliary, pattern, is not present. There is no evidence of either cavitary lung nodule or consolidation. 
Which of the following represents the next, most appropriate step for the assessment of the findings at chest radiography?

1. ${ }^{18}$ FDG-PET scan

2. Bilateral, frontal shallow $\left(5^{\circ}\right)$ oblique images to distinguish true lung nodule from artifact or a chest wall lesion

3. Obtaining prior thoracic imaging studies for comparison to determine if the solitary nodule is stable over time

4. Thoracic CT using a nodule enhancement protocol

5. Unenhanced thoracic CT 


\section{Correct! \\ 3. Obtaining prior thoracic imaging studies for comparison to determine if the solitary nodule is stable over time}

All of the options listed are appropriate measures used for the assessment of indeterminate solitary nodules. However, the first step in the evaluation of an indeterminate solitary pulmonary nodule should be comparison to prior thoracic imaging studies in the attempt to document long-term stability. As a general rule, documentation of long-term stability (usually 2 years or more) of an indeterminate nodule detected at chest radiography is sufficient to dismiss the nodule as benign without need for further exposure to ionizing radiation or the expense incurred by additional testing. If no such priors are available, or the nodule is new compared to older thoracic imaging studies, then the other methods listed are often employed to assess the nodule and drive the probability of malignancy either high enough to warrant intervention or low enough to safely permit observation.

No prior thoracic imaging studies were available for comparison, so the patient underwent bilateral, frontal, shallow $\left(5^{\circ}\right)$ oblique radiographs to distinguish a true lung nodule from either superimposition artifact or a chest wall abnormality simulating a pulmonary nodule.

Which of the following statements regarding this imaging study is most accurate?

1. The bilateral, frontal, shallow $\left(5^{\circ}\right)$ oblique radiographs are too technically suboptimal to allow reliable interpretation

2. The bilateral, frontal, shallow $\left(5^{\circ}\right)$ oblique radiographs fail to show the nodule, suggesting artifact as the cause of the appearance of a nodule at the original chest radiograph

3. The bilateral, frontal, shallow $\left(5^{\circ}\right)$ oblique radiographs show a tubular opacity leading to the nodule, suggesting that the nodule represents an arteriovenous malformation

4. The bilateral, frontal, shallow $\left(5^{\circ}\right)$ oblique radiographs show that the nodule "moves with" the ribs, confirming a chest wall origin for the nodule

5. The bilateral, frontal, shallow $\left(5^{\circ}\right)$ oblique radiographs show that the nodule "does not move" with the ribs, confirming a lung origin for the nodule 


\section{Correct!}

\section{The bilateral, frontal, shallow $\left(5^{\circ}\right)$ oblique radiographs show that the nodule "does not move" with the ribs, confirming a lung origin for the nodule}

The image on the left (Figure 1B) is a "right anterior oblique image- note how the right clavicle is projected across midline, indicating that the patient's right chest has moved anteriorly. The other image (Figure $1 \mathrm{C}$ ) is a left anterior oblique image- note how the patient's left clavicle crosses midline, indicating that the left chest has moved anteriorly. The nodule is visible in both projections; therefore it is not artifactual, and image quality is technically adequate. Note that on Figure $1 \mathrm{~A}$, the nodule projects between the $3^{\text {rd }}$ and $4^{\text {th }}$ anterior ribs, whereas on Figure $1 \mathrm{~B}$ and $1 \mathrm{C}$ the nodule projects directly over the $4^{\text {th }}$ anterior rib and then partially overlies the third anterior rib, respectively. The nodule also shows an inconsistent relationship to the posterior ribs. Because of this inconstant relationship to the ribs, the nodule must not originate from the chest wall, and instead resides within the lung. No evidence of a tubular morphology to suggest arteriovenous malformation is present.

Because the oblique chest radiographs confirmed the presence of an indeterminate lung nodule, thoracic CT (Figure 3) was performed.

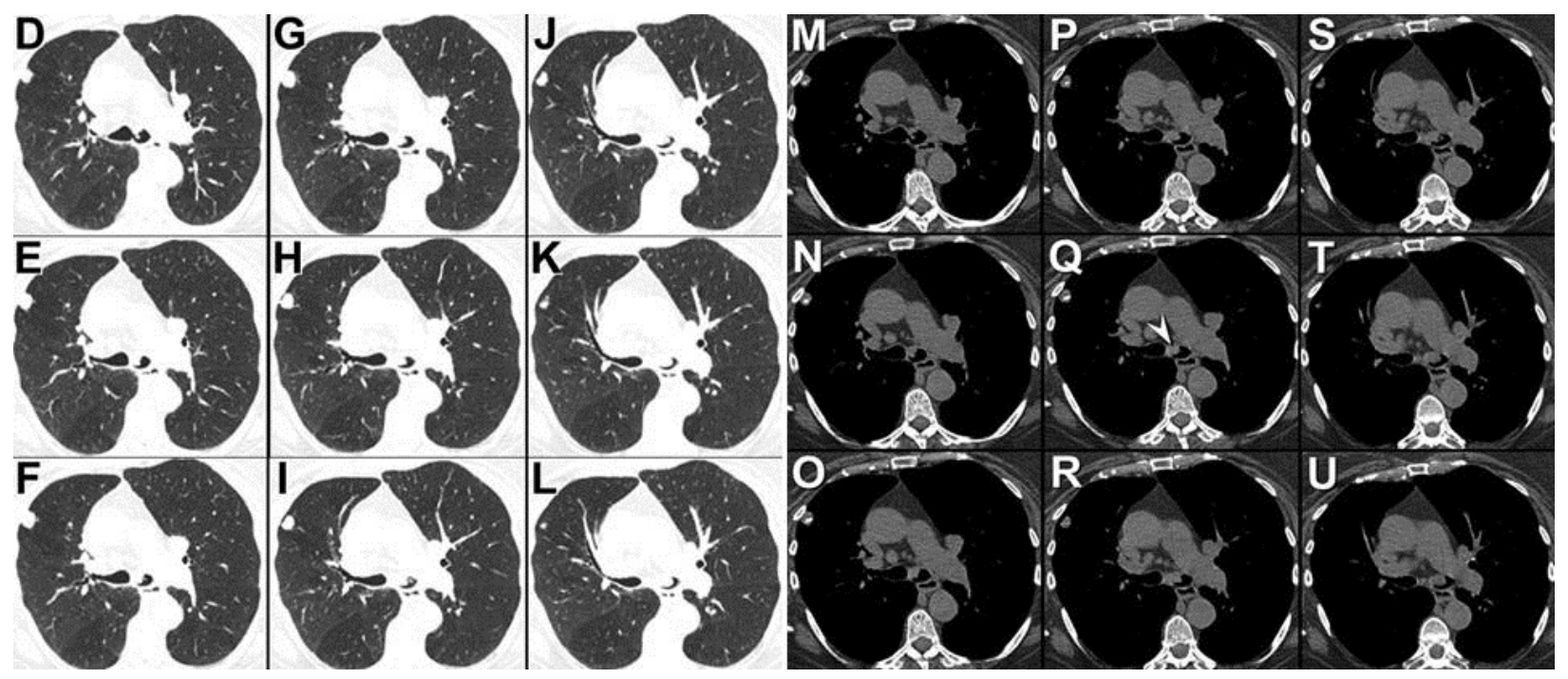

Figure 3. Thoracic CT displayed in lung windows (D- L) and soft tissue windows (M-U).

Which of the following statements regarding this CT examination is most accurate?

1. The thoracic CT shows a diffuse fibrotic process

2. The thoracic CT shows multifocal basal predominant bronchiectasis

3. The thoracic CT shows that the nodule at chest radiography is non-calcified and remains indeterminate

4. The thoracic CT shows that the nodule contains fat, consistent with hamartoma

5. The thoracic CT shows that the nodule is partially calcified but also reveals a left mainstem bronchial lesion 


\section{Correct!}

5. The thoracic CT shows that the nodule is partially calcified but also reveals a left mainstem bronchial lesion

The thoracic CT confirms the nodule resides in the subpleural region of the right upper lobe and is partially calcified (Figure 4).

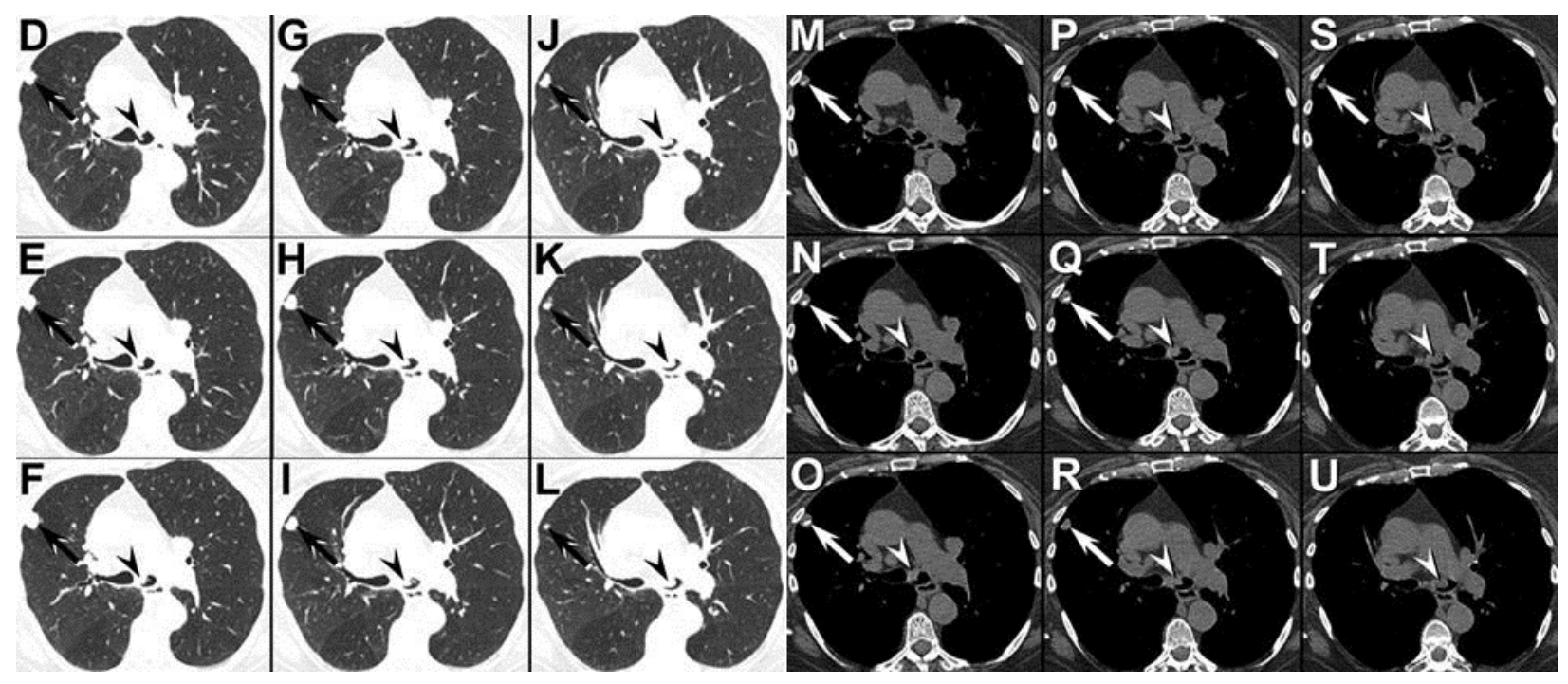

Figure 4. The peripheral right lung nodule (arrows) is circumscribed and partially calcified, which suggests a benign etiology. However, the thoracic CT also shows a polypoid, lobulated lesion (arrowheads) arising from the medial aspect of the left mainstem bronchus.

There is no evidence of low attenuation within the nodule to indicate fat to suggest the diagnosis of hamartoma. There is no evidence of bronchiectasis or fibrotic lung disease. The thoracic CT does also show a polypoid lesion residing within the left mainstem bronchus (Figure 4, arrowheads).

Which of the following represents the next most appropriate step for the evaluation of this patient?

1. ${ }^{18}$ FDG-PET scan

2. ${ }^{68} \mathrm{Ga}$-citrate scan

3. Flexible fiberoptic bronchoscopy

4. Percutaneous transthoracic needle biopsy

5. Video-assisted thoracoscopic biopsy 


\section{Correct! \\ 3. Flexible fiberoptic bronchoschopy}

The right lung nodule is at least partially calcified and therefore likely, although not definitely benign. Therefore, a conservative, observational approach to the management of this lesion is appropriate. Instead, attention turns towards the incidentally discovered airway lesion. The lesion resides within the left mainstem bronchus and is not accessible via either percutaneous transthoracic needle biopsy or video-assisted thoracoscopic biopsy. ${ }^{68} \mathrm{Ga}$-citrate (gallium) scanning would not provide managementaltering information. If the lesion shows active ${ }^{68} \mathrm{Ga}$ uptake, the diagnosis of carcinoid tumor would be a distinct possibility, but tissue sampling would nevertheless be required. The absence of ${ }^{68} \mathrm{Ga}$ tracer uptake within the lesion would not exclude the possibility of an aggressive lesion and tissue sampling is still required. Similarly, the presence of active tracer utilization at ${ }^{18}$ FDG-PET scanning would raise the possibility of an aggressive intervention, but would be non-specific as regards etiology, still necessitating a tissue diagnosis. The lack of tracer uptake at ${ }^{18}$ FDG-PET would not preclude the need for a tissue diagnosis; both benign and malignant endobronchial lesions may, or may not, show increased tracer utilization at ${ }^{18}$ FDG-PET.

Flexible fiberoptic bronchoscopy with biopsy was performed (Figure 5).

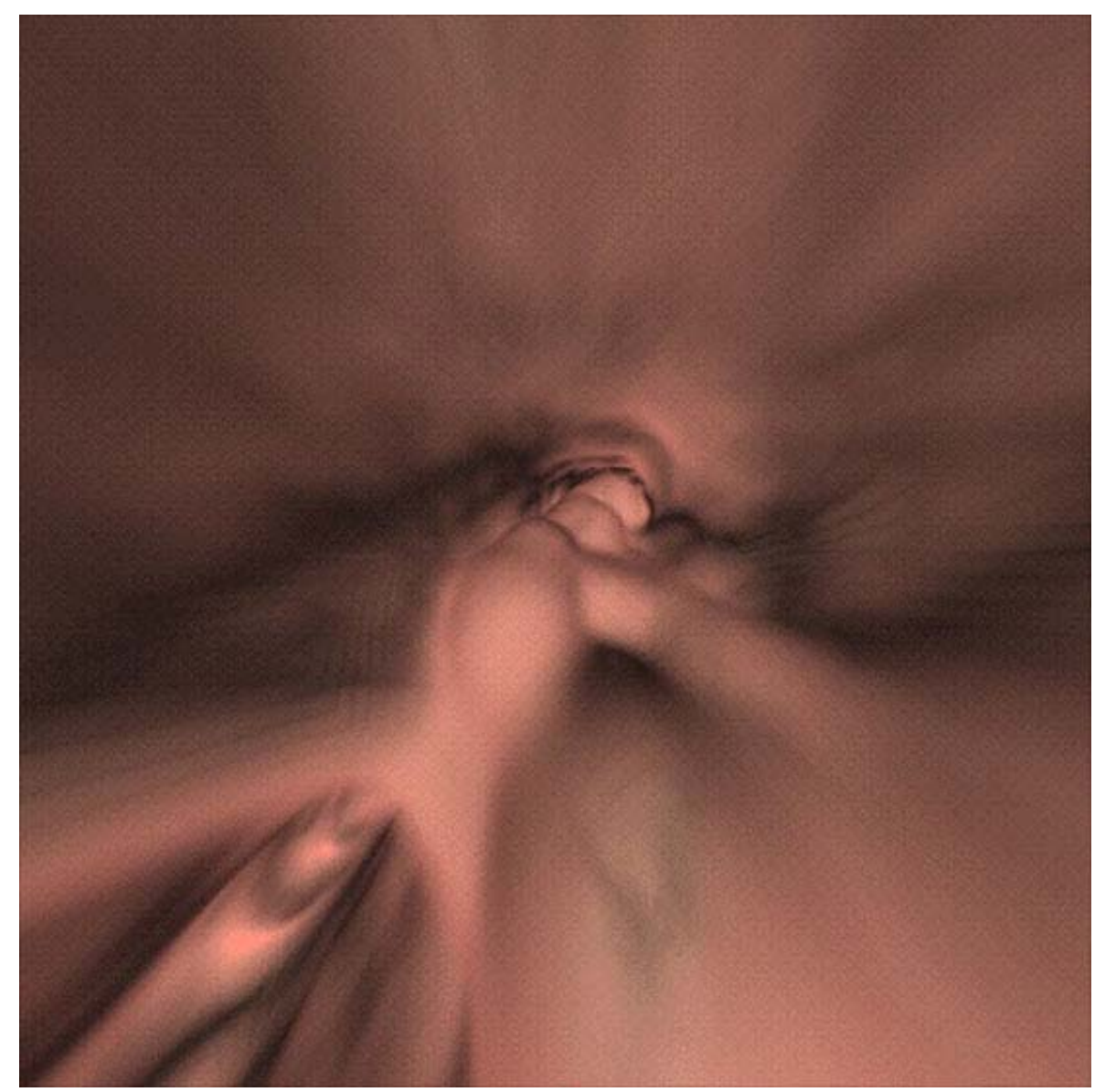

Figure 5. Virtual bronchoscopy provides the endoluminal perspective of this lesion. 
Biopsy material showing CD20+ B-lymphocytes co-expressing bcl-2 and lacking expression for CD3, CD10, bcl-6, and cyclin D1, and without evidence of kappa or lambda immunoperoxidase staining, supporting the diagnosis of lymphoma

Diagnosis: Extranodal marginal zone lymphoma arising from mucosa-associated lymphoid tissue (MALT).

\section{References}

1. William J, Variakojis D, Yeldandi A, Raparia K. Lymphoproliferative neoplasms of the lung: a review. Arch Pathol Lab Med 2013; 137(3):382-391. [CrossRef] [PubMed]

2. Zhang WD, Guan YB, Li CX, Huang XB, Zhang FJ. Pulmonary mucosa-associated lymphoid tissue lymphoma: computed tomography and ${ }^{18} \mathrm{~F}$ fluorodeoxyglucosepositron emission tomography/computed tomography imaging findings and followup. J Comput Assist Tomogr 2011; 35(5):608-613. [CrossRef] [PubMed]

3. Hare SS, Souza CA, Bain G, Seely JM, Frcpc, Gomes MM, Quigley M. The radiological spectrum of pulmonary lymphoproliferative disease. Br J Radiol 2012; 85(1015):848-864. [CrossRef] [PubMed]

4. Do KH, Lee JS, Seo JB, Song JW, Chung MJ, Heo JN, Song KS, Lim TH. Pulmonary parenchymal involvement of low-grade lymphoproliferative disorders. J Comput Assist Tomogr 2005; 29(6):825-830. [CrossRef] [PubMed]

5. Michael CW, Richardson PH, Boudreaux CW. Pulmonary lymphoma of the mucosaassociated lymphoid tissue type: Report of a case with cytological, histological, immunophenotypical correlation, and review of the literature. Ann Diagn Pathol 2005; 9(3):148-152. [CrossRef] [PubMed] 